\title{
A proposal of structure and control overcoming conducted electromagnetic interference in a buck converter
}

\author{
Zakaria M'barki, Kaoutar Senhaji Rhazi, Youssef Mejdoub \\ Laboratory of Networks, Computer Science, Telecommunication, Multimedia (RITM), CED Engineering Sciences, \\ Higher School of Technology, Hassan II University, Casablanca, Morocco
}

\begin{tabular}{l} 
Article Info \\
\hline Article history: \\
Received Jul 15, 2021 \\
Revised Jan 19, 2022 \\
Accepted Jan 26, 2022 \\
\hline
\end{tabular}

Keywords:

Conducted EMI

Power electronics

Random PWM

Soft-switching

\begin{abstract}
This paper describes modelling a step-down chopper designed for a photovoltaic application to study its performance of electromagnetic compatibility (EMC) in conducted mode. The EMC measurements attended at this stage have shown that the buck converter is a harmful device for its electromagnetic environment. Indeed, the fast-switching operation leads to higher levels of conducted and radiated electromagnetic interference (EMI) and subsequently facilitates the electromagnetic coupling with the surrounding environment. In this sense, we present a new proposal of structure and control based on a combination of two techniques, namely pseudorandom modulation and soft switching, thus minimizing the conducted electromagnetic emissions at the source. The usefulness of this new strategy, evaluated through a comparative study, lies in its efficiency compared to the classical PWM method, allowing both to reduce the rise of current and voltage gradients and spread the electromagnetic spectrum over a wide frequency range. The simulation results prove that a significant gain in EMC has been reached.
\end{abstract}

This is an open access article under the CC BY-SA license.

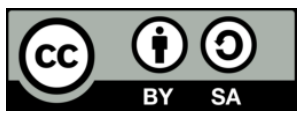

\section{Corresponding Author:}

Zakaria M'barki

RITM Laboratory, Higher School of Technology, Hassan II University of Casablanca

Km 7 El Jadida Road-r.p.8, B.P. 20000, Casablanca-Morocco

Email: mbarki.ensem@gmail.com

\section{INTRODUCTION}

Over the last decade, photovoltaic energy has become more and more widespread. As a result, conversion systems such as choppers and inverters have become necessary to adapt to different technologies and environments [1], [2]. However, these semiconductor-based power converters, ubiquitous in various fields, operate polluting due to the fast switching. In general, the switching operation exhibits quasi-trapezoidal waveforms (voltages and currents). Analyzing these waveforms becomes essential for proper modeling noise sources and propagation paths in converter systems [3]. In this perspective, our subject is interested in studying the conducted electromagnetic stresses [4] in a buck converter dedicated to a photovoltaic application to minimize them as much as possible. Indeed, the power switch used, such as the metal oxide semiconductor field effect transistor (MOSFET), generally presents two transient switching phases: turn-off and turn-on. High current and voltage differentials are generated during these phases, which subsequently induce disturbing emissions propagating by conduction and radiation in the frequency band between $(150 \mathrm{kHz}, 1 \mathrm{GHz})$ [5]. Again, the increase in frequency synchronized with the evolution of power electronic components has made it possible to increase the power density $(\mathrm{kW} / \mathrm{kg})$ and reduce manufacturing costs. However, it leads to a reduction in efficiency (higher switching losses) and an increase in electromagnetic pollution (constraints on the MOSFET and translation of the spectrum towards HF). To remedy this, several methods mentioned in the 
literature have been discussed. First of all, the resonant switching method [6], also called soft switching, is occurred either at zero voltage [7] or zero current [8]. The zero-voltage switching quasi-resonant buck converter (ZVS-QRC) is one of the most widely used advanced ZVS schemes. Its principle is to add some extra elements (inductor, capacitor, or diode) to the main switch to overcome the already expected drawbacks of the hard switching method for a conventional buck converter. Thus, the power losses due to switching are considerably reduced on the one hand, and on the other hand, voltage or current gradients are attenuated. That ensures a reduction in conducted electromagnetic interference and subsequently contributes to a good electromagnetic compatibility (EMC) gain.

In the same vein, other solutions have proven to be quite effective, according to previous work dedicated to this field of EMC. The most widely used technique is filtering [9], [10], which reduces or eliminates the EMI noise generated by power converters. However, when size is a significant concern, another option should be to minimize conducted emissions, namely pseudo-random modulation [11]. Its effectiveness lies in how electromagnetic noise levels are reduced while dispersing harmonic energy over a wide frequency range [12], [13]. This method is easy to implement at the control level because the structure of the conversion system is preserved. At this stage, there are two types of implementations: a logic implementation and a digital implementation via specific circuits such as field-programmable gate array (FPGA) and digital signal processing (DSP). In this context, the procedures widely used to produce this type of pseudo-random modulation are mainly focused on the use of a linear feedback shift register (LFSR) [14], a linear congruential generator (LGC) [15], or a chaotic recursive sequence [16], [17].

In order to better meet the EMC standards, a promising approach based on an appropriate combination of pseudo-random modulation and resonant switching techniques has been developed, encompassing the advantages of both techniques already provided. Comparison based on the spectral content of electromagnetic disturbances for different methods shows the effectiveness of this new method compared to the others. The present work studies a DC-DC conversion system used in photovoltaic applications, namely the step-down chopper. As it is a polluting source of its electromagnetic environment, we will mention the different methods to optimize and reduce its electromagnetic emissions, especially those carried out to comply with the prescribed EMC rules [18]. Finally, a brief conclusion is included.

\section{STUDY \& MEASUREMENT OF CONDUCTED DISTURBANCES OF A BUCK CONVERTER}

A static step-down converter of duty cycle $\alpha$ is interposed between the load R_L and the photovoltaic generator represented by a DC voltage source $V_{\text {in }}$ which is assumed to be a constant input. To study the characteristics of the switches considered for this application, we have chosen a chopper model consisting of a freewheeling diode (HFA25TB60) and a MOSFET transistor (IRFP460) operating at very high frequencies for power applications of the order of $1 \mathrm{KW}$. The MOSFET is controlled by a logic signal $V_{G S}$ of fixed frequency $(100 \mathrm{kHz})$ and duty cycle $\alpha=0.5$. The latter is generated by the principle of deterministic pulse width modulation (DPWM), i.e., based on a comparison between a reference modulating signal $V_{\text {ref }}$ and a carrier $V_{\text {tri }}$ (Figure 1).

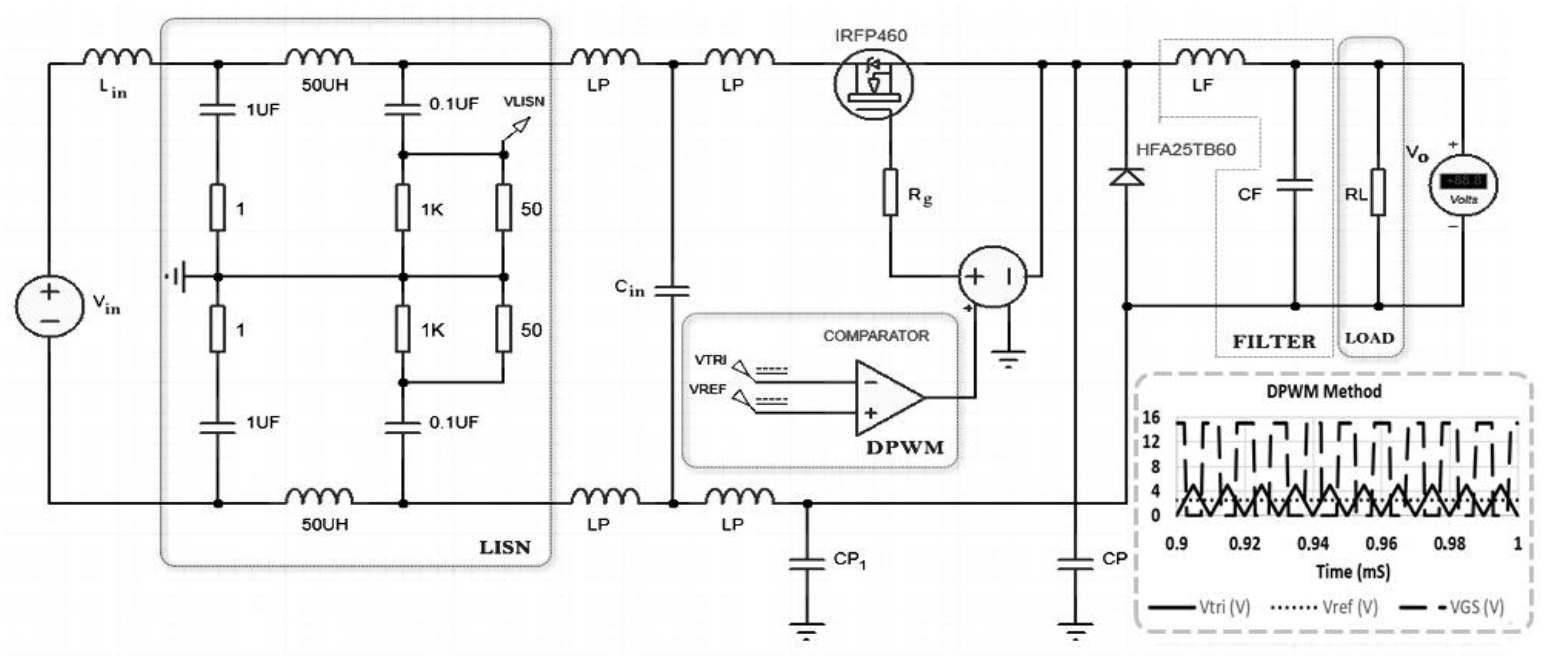

Figure 1. The structure of the serial chopper used 
The LC output filter of the buck converter is used to limit the maximum current ripple $\left(\Delta I_{L}=20 \%\right)$ in the inductor and the maximum voltage ripple $\left(\Delta V_{O}=2 \%\right)$ across the load (Figure 2). The following expressions give the calculation of the components of this filter:

$$
L_{F}=\frac{\left(V_{i n}-V_{O}\right) \mu T_{S}}{\Delta I_{L}} ; C_{F}=\frac{\Delta I_{L} T_{S}}{8 . \Delta V_{O}}
$$

This buck converter is designed for the following specifications listed in Table 1.

Table 1. Simulation parameters

\begin{tabular}{lc}
\hline \multicolumn{1}{c}{ Parameter } & Value \\
\hline Input voltage $V_{i n}$ & $100 \mathrm{~V}$ \\
Output voltage $V_{O}$ & $50 \mathrm{~V}$ \\
Load resistance $R_{L}$ & $24 \Omega$ \\
Switching frequency $f_{S}=\frac{1}{T_{S}}$ & $100 \mathrm{kHz}$ \\
Inductance $L_{F}$ & $600 \mu \mathrm{H}$ \\
Capacitance $C_{F}$ & $520 \mathrm{nH}$ \\
Conversion ratio $\mu=\frac{V_{O}}{V_{i n}}$ & $50 \%$ \\
\hline
\end{tabular}
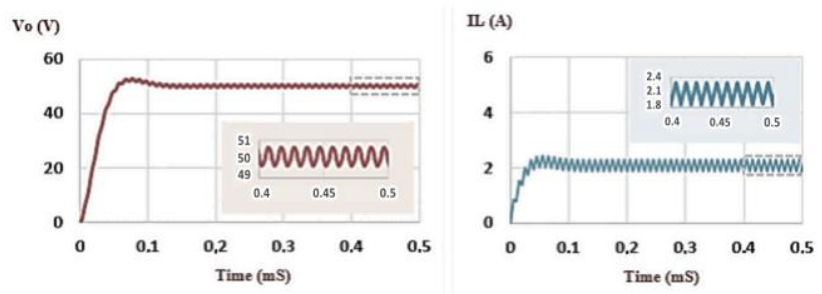

Figure 2. Steady-state waveforms $\left(I_{L}\right.$ and $\left.V_{O}\right)$ for the serial chopper

EMI are harmful disturbances that affect neighboring electrical circuits due to electromagnetic conduction or electromagnetic radiation from an external source. These disturbances can interrupt or even degrade the actual performance of circuits. EMI is classified into two categories: conducted and radiated. Conducted EMI is caused by physical contact with metallic conductors, while radiated EMI is caused by induction (without physical contact with the conductors). The Figure 3 (a) show the different types of EMI.

In this section, we will only focus on conducted EMIs. In order to be able to quantify them, a measuring device has been developed for this purpose. This instrument, which is prescribed in the EMC standards, is called a line impedance stabilization network "LISN" in Figure 1 and is a tool for measuring conducted EMI [19]; it is similar to a filter that is inserted between the device under test and the network supplying the energy. Its function is to isolate the network on which common mode and differential mode disturbances may exist in Figure 3 (b) from the equipment under test (step down chopper).

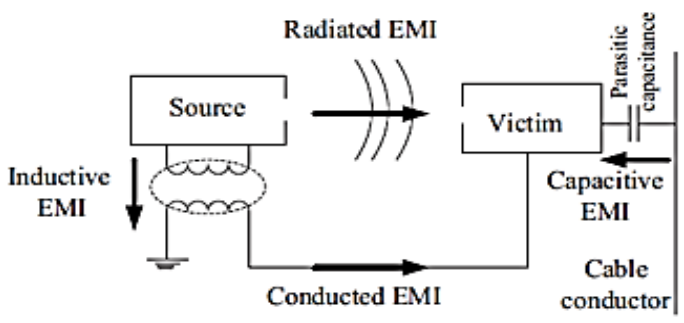

(a)

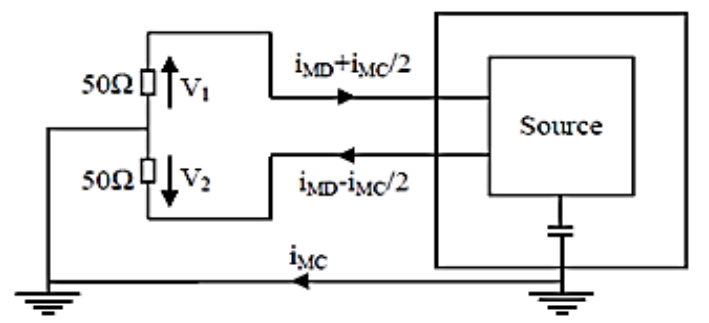

(b)

Figure 3. Electromagnetic emissions with (a) EMI classes and (b) principle of measurement of conducted disturbances

\section{METHODS, SIMULATION RESULTS AND DISCUSSION}

\subsection{Soft switching}

Controlled switching of a semiconductor can pose stringent requirements for electromagnetic compatibility. Indeed, conducted emission is considered a significant problem on most conventional power electronics systems. The slight variations in current and voltage that the cell undergoes during the switching phase are fundamental sources of disturbance. In addition, the high frequency of static converters leads to a significant increase in switching losses. This type of switching is called hard switching.

In order to overcome these drawbacks, a combination of power electronics topologies and appropriate switching strategies is proposed. This is achieved by incorporating resonant circuits into conventional converter topologies to create zero voltage switching (ZVS) or zero current switching (ZCS) conditions for semi- 
conductor switches. This type of switching is called soft switching [20], as opposed to DPWM or so-called hard switching. ZVS and ZCS switching topologies specifically use resonance techniques to force the voltage or current in a semi-conductor switch to zero, thereby eliminating or reducing switching losses. These converters have the same power topologies as conventional hard-switching converters, except that they use an LC resonant circuit around the semi-conductor switches. Depending on the location of the ZVS or ZCS resonant circuit elements, turn-on or turn-off transitions can be created. Typically, in ZCS converters, a resonant inductor placed in series with the semi-conductor switch is used to resonate the current through the switch to zero on opening and limit the $\frac{\mathrm{di}}{\mathrm{dt}}$ on closing. Generally, in ZVS converters, a resonant capacitor placed in parallel with the semiconductor switch is used to resonate the voltage across the switch to zero on closure and limit the $\frac{\mathrm{dv}}{\mathrm{dt}}$ on opening. In this paper, the focus will be on the ZVS method and its effectiveness in reducing EMIs. The following properties characterize the design of buck quasi-resonant converters operating at zero voltage:

- Normalized resonance frequency: $f_{n}=\frac{f}{f_{r}}$ with $f_{r}=\frac{1}{2 \pi \sqrt{L_{R} C_{R}}}$

- Normalized characteristic impedance: $Z_{n}=\sqrt{\frac{L_{R}}{C_{R}}}$

- Normalized load resistance: $\mathrm{Q}=\frac{R_{L}}{Z_{n}}$

- Conversion ratio : $\mu=\frac{V_{\text {out }}}{V_{\text {in }}}$

Figure 4 shows the schematic of the buck DC/DC converter under study. This schematic which operates on ZVS condition as shown in Figure 4 (a) can be divided into four operating modes as shown in Figure 4 (b), which are dependent on the state of the main switch and the freewheeling diode. It is assumed that the inductance and capacitance of the output filter are much larger than the inductance and capacitance of the resonance, and therefore, the output current and voltage can be considered as pure DC parameters equal to $I_{O}$ and $V_{O}$, respectively.

- Mode I $\left(0 \leq \mathrm{t}<t_{1}\right)$

At the beginning of this mode, the switch $\mathrm{S}$ is turned off, diverting the current $i_{L R}$ into the resonant capacitor $C_{R}$. The voltage across the capacitor, initially zero, begins to charge with a current equal to $I_{O}$. The equivalent circuit in this mode is shown in Figure 4 (b-1) This gives:

$$
V_{C R}=\frac{I_{O}}{C_{R}} \mathrm{t} ; V_{D}=V_{i n}-V_{C R}
$$

The duration of this mode is given by: $\tau_{1}=t_{1}=\frac{V_{\text {in }} C_{R}}{I_{0}}$

- Mode II $\left(t_{1} \leq \mathrm{t}<t_{2}\right)$

This mode starts when diode $\mathrm{D}$ becomes forward biased. During this mode, capacitor $C_{R}$ and inductor $L_{R}$ together form a resonant circuit, and at the end of this mode, the voltage $V_{C R}$ attempts to reverse, which causes diode $D_{R}$ to conduct. The diode $D_{R}$ across the capacitor $C_{R}$ prevents the voltage $V_{C R}$ from becoming negative. The equivalent circuit in this mode is shown in Figure 4 (b-2). These yields:

$$
i_{L R}=I_{O} \cos w_{r}\left(\mathrm{t}-t_{1}\right) ; V_{C R}=V_{i n}+I_{O} Z_{n} \sin w_{r}\left(\mathrm{t}-t_{1}\right) ; I_{O} \geq \frac{V_{i n}}{Z_{n}}
$$

The length of this mode is given by: $\tau_{2}=t_{2}-t_{1}=\frac{1}{W_{r}}[\pi+\gamma]$ avec $\gamma=\sin ^{-1}\left(\frac{V_{\text {in }}}{Z_{n} I_{O}}\right)$

- Mode III $\left(t_{2} \leq \mathrm{t}<t_{3}\right)$

This mode begins as soon as switch $\mathrm{S}$ is turned on. The equivalent circuit for this mode is shown in Figure 4 (b-3). During this mode, diode D remains forward biased and conductive. Furthermore, in this mode, the current $i_{L R}$ increases linearly from $t_{3}$ to $t_{4}$ until it reaches the value of $I_{O}$. The following equations are obtained:

$$
i_{L R}=\frac{V_{\text {in }}}{L_{R}}\left(t-t_{2}\right)-I_{O} \cos (\gamma) ; V_{C R}=0
$$

The period of this mode is given by: $\tau_{3}=t_{3}-t_{2}=\frac{I_{0} L_{R}}{V_{\text {in }}}(1+\cos \gamma)$.

- Mode IV $\left(t_{3} \leq \mathrm{t}<T_{s}\right)$

This mode starts when the resonant inductance current $i_{L R}$ becomes equal to the output current $I_{O}$ and, as a result, diode $\mathrm{D}$ becomes reverse biased and stops conducting. Therefore, in this mode, only switch $\mathrm{S}$ is 
conducting and transferring power to the load. The equivalent circuit for this mode is shown in Figure 4 (b-4). The following equations are derived.

$$
i_{L R}=I_{O} ; V_{D}=V_{i n} ; V_{C R}=0
$$

This mode will end at $t_{4}=T_{s}$, and its time length is given by: $\tau_{4}=T_{s}-\tau_{3}-\tau_{2}-\tau_{1}$

We will now discuss the simulation results of the switch voltages and currents as shown in Figure 5. The effect of the resonant circuit is manifest on the EMI stresses as the slope of the switch voltage $V_{D S}$ is well reduced compared to the conventional method as shown in Figure 6, the switching losses are considerably reduced.

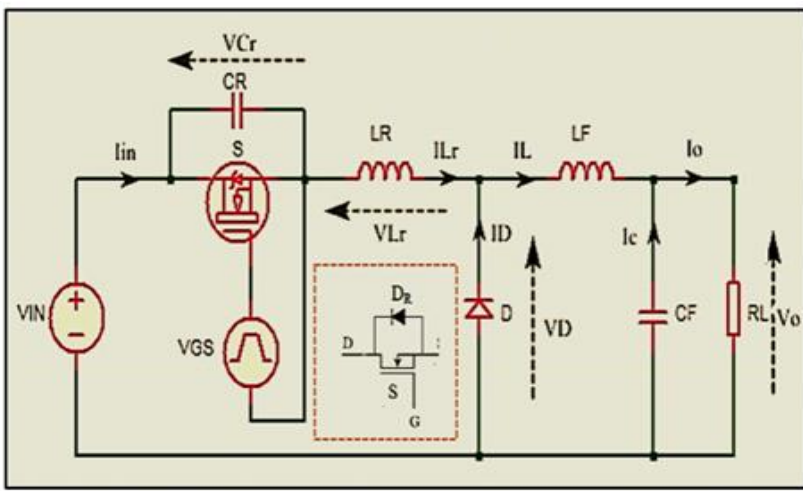

(a)

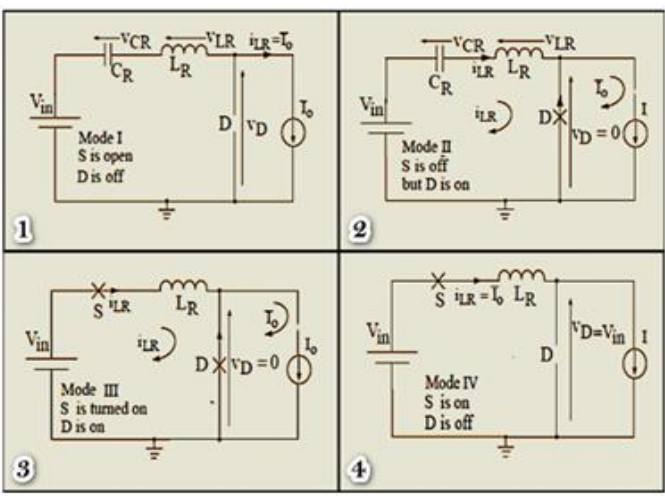

(b)

Figure 4. Schematic of the buck quasi-resonant converter operated under with (a) zero voltage switching and (b) its equivalent circuits for operating modes
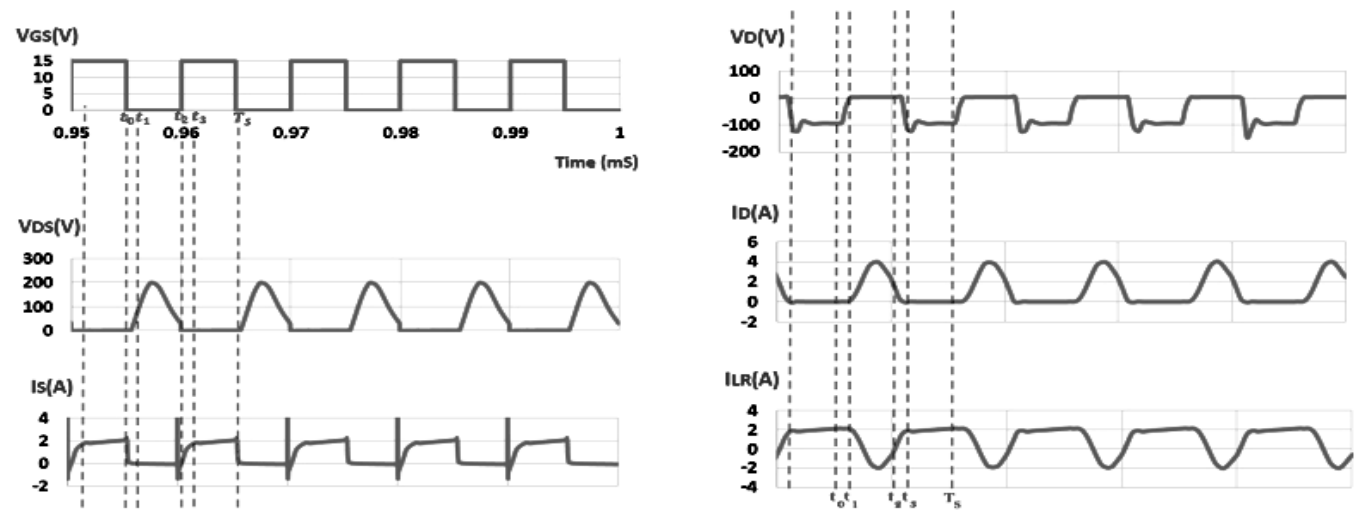

Figure 5. Voltage and current for ZVS method

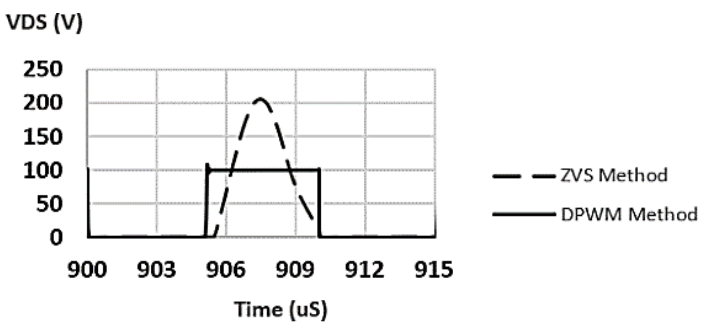

Figure 6. $V_{D S}$ Voltage slope for DPWM and ZVS methods 
By using the concept of energy conservation, we get the expression of the voltage gain:

$$
\mu=1-\frac{f_{n}}{2 \pi}\left(\frac{Q}{2 \mu}+\pi+\sin ^{-1}\left(\frac{Q}{\mu}\right)+\frac{\mu}{Q}\left(1+\sqrt{1-\left(\frac{Q}{\mu}\right)^{2}}\right)\right.
$$

This yields the expression for the duty cycle:

$$
\mathrm{D}=1-\frac{f_{n}}{2 \pi}\left(\frac{Q}{\mu}+\pi+\sin ^{-1}\left(\frac{Q}{\mu}\right)\right)
$$

The plotting of the conversion ratio $\mu$ as a function of the normalized frequency is achieved for different loads Q as shown in Figure 7. A numerical analysis method of Newton Raphson has been applied. Here it can be deduced that in the case of the ZVS control, the output voltage depends on the control frequency.

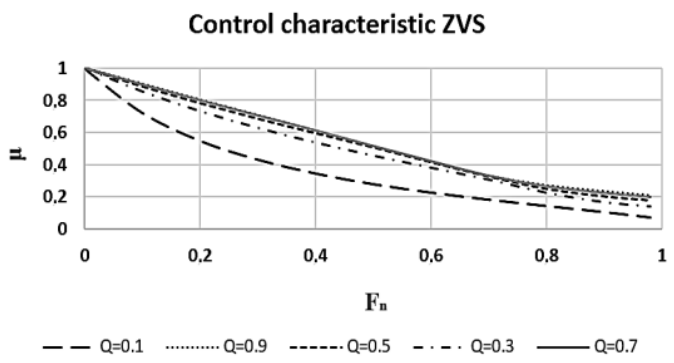

Figure 7. Conversion ratio characteristic according to the normalized load

Based on the study of the quasi-resonant buck converter in the previous paragraph, the following results can be given, assuming that we take $\mathrm{Q}=\mu=0.5$ :

The resonant frequency: $f_{r}=\frac{3(1+\pi) f_{S}}{4 \pi(1-\mu)}$

The duty cycle: $\mathrm{D}=1-\frac{f_{n}}{4 \pi}(3 \pi+2)$

These formulas calculate the resonant inductor and capacitor:

$$
L_{R}=\frac{Z_{n}}{2 \pi f_{r}} ; C_{R}=\frac{1}{2 \pi f_{r} Z_{n}}
$$

As shown in Table 2 summarizes what has been described above.

Table 2. The buck ZVS-QRC converter parameters

\begin{tabular}{lc}
\hline \multicolumn{1}{c}{ Component } & Normalized value \\
\hline Inductance $L_{R}$ & $39 \mu \mathrm{H}$ \\
Capacitance $C_{R}$ & $15 \mathrm{nH}$ \\
Resonant frequency $f_{r}$ & $197.75 \mathrm{kHz}$ \\
Duty cycle D & $\sim 54 \%$ \\
\hline
\end{tabular}

\subsection{Pseudo random modulation}

Its principle lies in generating a chaotic binary sequence of 0 and 1 (PRBS) from linear feedback shift registers. The theory [21] behind these devices is based on algebraic computation in the Galois body as shown in Figure 8 (a). The resulting binary sequence lasts $\mathrm{L}=\left(2^{N}-1\right)^{*} T_{C L K}$. Where $\mathrm{N}$ is the toggle number, and $T_{C L K}$ is the clock period. If $\mathrm{N}$ becomes large, then the observation of one of the outputs of the $\mathrm{N}$ flip-flops reveals a random series of 1 and 0 . The repetition period $\mathrm{L}$ is vast, which justifies the name pseudo-random sequence. After that, we take in our work $f_{C L K}=100 \mathrm{kHz}$ and $\mathrm{N}=8$ in Figure 8 (b); thus, we will have a binary sequence of $\mathrm{L}=255$.

This technique will be used to perform a pseudo-random modulation control. A multiplexer whose address input is the random sequence of 0 and 1 will direct two types of input signals of triangular form in the opposite phase to the output, which will be considered a carrier for our control system. Next comes the operation of comparing the reference signal $V_{\text {ref }}$ to this random carrier $V_{P}$ to generate the switch control signal as shown in Figure 9. 
This resulting control signal has a pseudo-random pulse position with a constant duty cycle and frequency switching. That is called random pulse position modulation (RPPM) [22], [23]. Simulation analysis of this same signal in the frequency domain via the fast Fourier transform (FFT) enabled us to extract its spectrum and compare it with that derived from the DPWM method (Figure 10). As a result, it is found that the spectrum of the signal via the pseudo-random modulation method is well-distributed, thus reducing the harmonics peaks compared to the conventional DPWM method, whose spectrum contains odd-numbered harmonics with significant amplitudes.

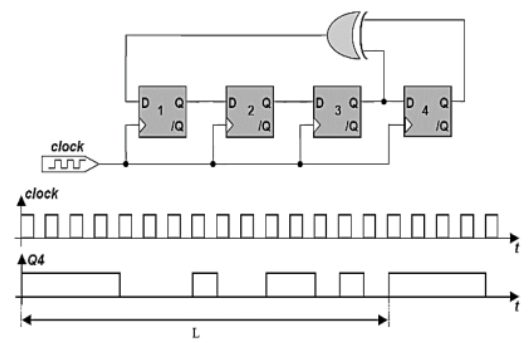

(a)

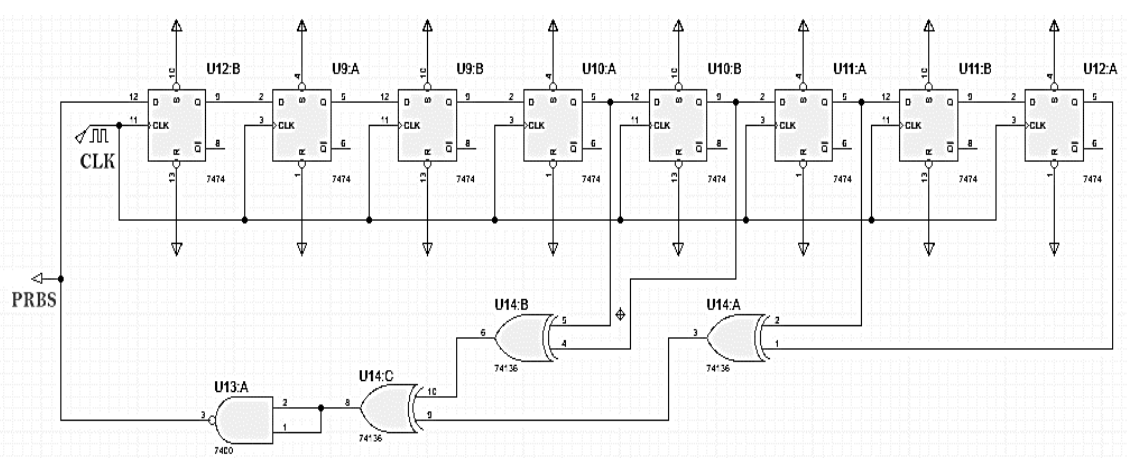

(b)

Figure 8. Pseudo-random binary sequence (PRBS) with (a) operating model and (b) PRBS generator circuit

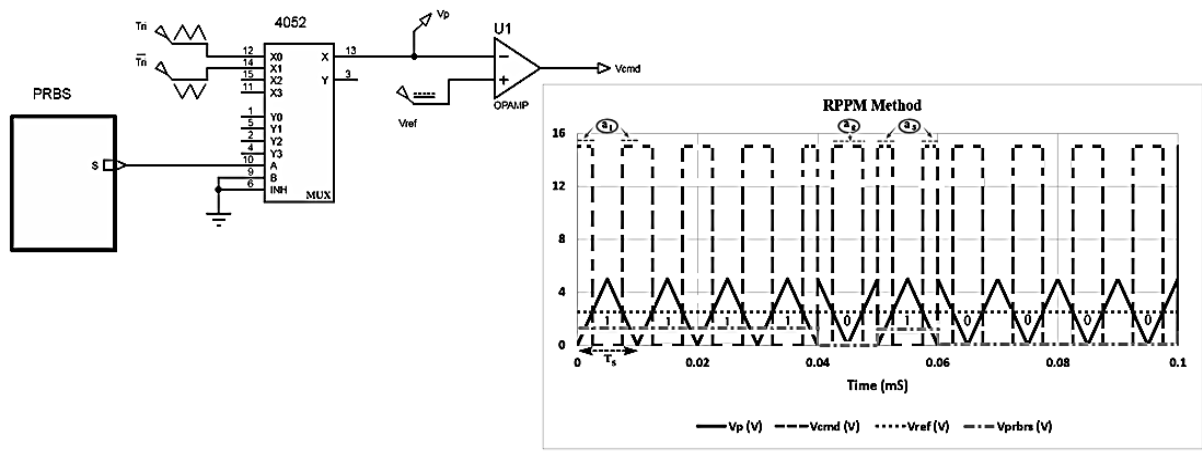

Figure 9. Structure of the control signal generator for RPPM method

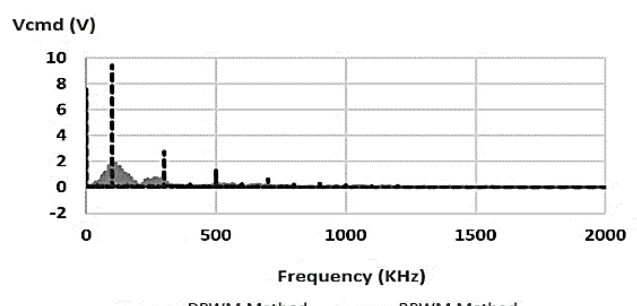

Figure 10. Spectrum of control signal for DPWM and RPPM methods 


\subsection{Conducted disturbance analysis for the different methods}

This part of research typically aims to quantify EMI in the conducted mode for a buck converter, and this for different approaches already mentioned. We opted for analysis in the time domain [24] instead of the frequency domain [25] for direct modeling of EMI. This simple analysis is performed using circuit simulation software (ISIS PROTEUS, SPICE, and PSIM). The spectrum of disturbances is then obtained by fast Fourier transform (FFT).

Indeed, the voltage measured at the terminals of the LISN is an image that reflects the disturbances in differential and common mode. To analyze it, we will be interested in extracting the spectral content it carries. The scheme we will adopt in the following is shown in Figure 11.

The main objective of the present study is to visualize the impact of these methods, respectively ZVSSwitching and RPPM, on the optimization of conducted electromagnetic emissions in the frequency range between $(150 \mathrm{kHz}, 30 \mathrm{MHz})$. Finally, a new method will be proposed that is based on a combination of these methods. By using simulation, the following EMI measurement results were collected in Figure 11.

Figure 11 (a) shows a direct comparison at the frequency spectrum level $\left(V_{\text {lisn }}\right)$ between the two soft and hard switching methods. In the frequency range between $(3 \mathrm{MHz}, 30 \mathrm{MHz})$, it is evident that the spectral content of the voltage $V_{l i s n}$ has decreased significantly. In a resonant converter, the master switches turn on and turn off in the ZVS condition, which reduces the switching gradients $\frac{\mathrm{dv}}{\mathrm{dt}}$ and $\frac{\mathrm{di}}{\mathrm{dt}}$. As a result, the softswitching converter significantly reduces EMI noise. Hence, it is of primary interest in terms of EMC. We will then analyze Figure 11 (b), which also shows a comparative spectral content of the voltage $V_{\text {lisn }}$ between two techniques, i. e., classical PWM (DPWM) and random (RPWM). The pseudo-random modulation employed is based on the randomization of the pulse position (RPPM). It can be seen that the spectral content distribution derived from the latter technique is extended uniformly over a wide frequency band. That will lead to an effective attenuation of harmonic amplitudes; in other words, an EMC gain of approximately $20 \mathrm{dBuV}$ compared to the DPWM technique can be achieved.

Finally, to benefit from the advantages of the two proposed methods, they are combined in a unique technique: The dual method. This method makes it possible to mix the two strategies without altering the level of the output voltage. As shown in Figure 11 (c), we can see that the spectrum is uniformly spread over an extensive frequency band with harmonics whose peaks have significantly been reduced. The results we have identified further explain the usefulness of this process for reducing conducted disturbances in the buck converter system. The EMC measurements show that the levels of conducted EMI are effectively reduced.

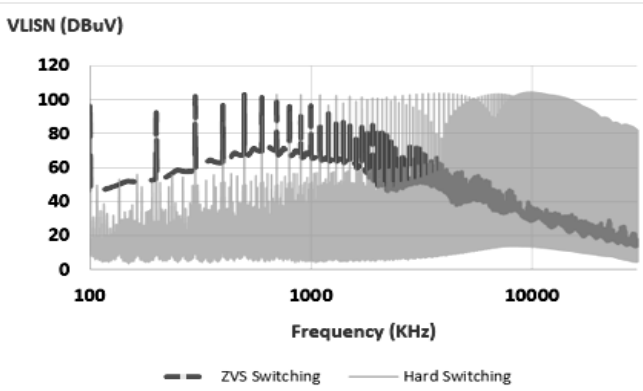

(a)

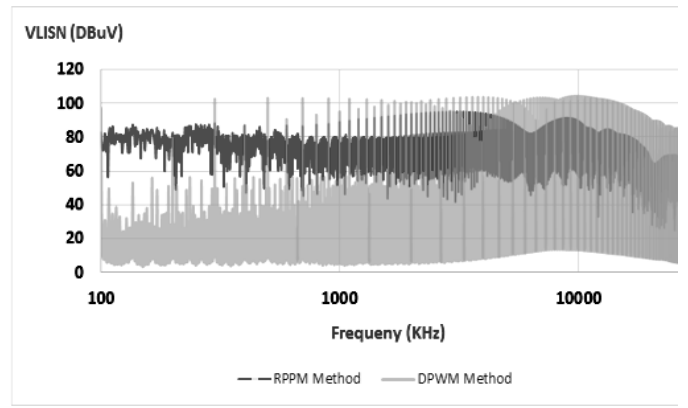

(b)

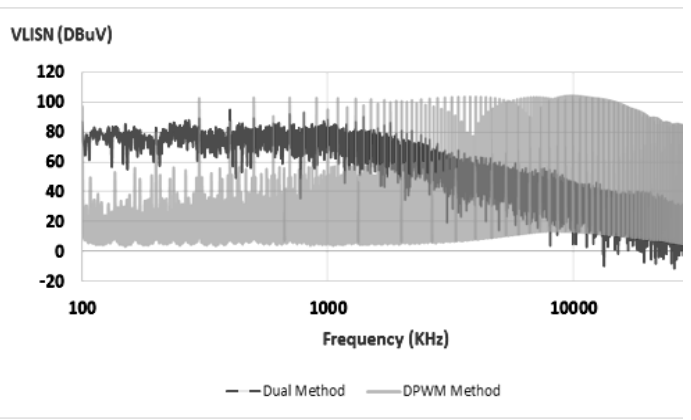

(c)

Figure 11. Spectral content of the voltage $V_{\text {lisn }}$ for (a) ZVS method, (b) RPPM method, and (c) dual method 


\section{CONCLUSION}

A new technique based on the combination of two methods has been discussed in this paper to reduce the adverse effects of conducted disturbances in static DC-DC converters. The first method uses resonant circuits to smooth the switching of turn on or off, thereby reducing the rate of voltage and the current rise and achieving significant EMC gain. The second is to use pseudo-random control (RPPM), which spreads the power spectrum of disturbances over a wide range of frequencies and thus reduces the amplitudes or peaks of harmonics that come from deterministic PWM control. An evaluation of the effectiveness of this new strategy based on the spectral analysis of conducted EMI has shown that the conducted emissions of the studied device are considerably reduced, and therefore, a meaningful gain in electromagnetic compatibility has been achieved.

\section{REFERENCES}

[1] A. Salman, A. Williams, H. Amjad, M. K. L. Bhatti and M. Saad, "Simplified modeling of a PV panel by using PSIM and its comparison with laboratory test results," IEEE Global Humanitarian Technology Conference (GHTC), 2015, pp. 360-364, doi: 10.1109/GHTC.2015.7343997.

[2] K. V. G. Raghavendra et al., "A Comprehensive Review of DC-DC Converter Topologies and Modulation Strategies with Recent Advances in Solar Photovoltaic Systems," Electronics, vol. 9, pp. 1-41, 2019, doi: 10.3390/electronics9010031.

[3] L. Fakhfakh, A. Alahdal, and A. Ammous, "Fast modeling of conducted EMI phenomena using improved classical models," AsiaPacific International Symposium on Electromagnetic Compatibility (APEMC), 2016, pp. 549-552, doi: 10.1109/APEMC.2016.7522795.

[4] A. Farhadi and A. Jalilian, "Modeling and Simulation of Electromagnetic Conducted Emission Due to Power Electronics Converters," International Conference on Power Electronic, Drives and Energy Systems, 2006, pp. 1-6, doi: 10.1109/PEDES.2006.344331

[5] R. Redl, "Power electronics and electromagnetic compatibility," PESC Record. 27th Annual IEEE Power Electronics Specialists Conference, 1996, pp. 15-21 vol.1, doi: 10.1109/PESC.1996.548553.

[6] F. C. Lee, "High-frequency quasi-resoant converter technologies," Proceedings of the IEEE, vol. 76, no. 4, pp. 377-390, 1988, doi: $10.1109 / 5.4424$.

[7] Y. Yinghua, G. Honglin, W. Xinhua, and T. Jinfei, "Study on Soft Switching Technology to Reduce Electromagnetic Interference of PWM Inverter,” Energy Procedia, vol. 17, pp. 384-390, 2012, doi: 10.1016/j.egypro.2012.02.110.

[8] K.-K. Chen, "A Novel Application of Zero-Current-Switching Quasiresonant Buck Converter for Battery Chargers," Mathematical Problems in Engineering, vol. 2011, ID. 481208, pp 1-16, 2011, doi: 10.1155/2011/481208.

[9] J. L. Kotny, T. Duquesne and N. Idir, "Design of EMI filters for DC-DC converter," IEEE Vehicle Power and Propulsion Conference, 2010, pp. 1-6, doi: 10.1109/VPPC.2010.5729047.

[10] W. Chen, W. Zhang, X. Yang, Z. Sheng and Z. Wang, "An Experimental Study of Common- and Differential-Mode Active EMI Filter Compensation Characteristics," IEEE Transactions on Electromagnetic Compatibility, vol. 51, no. 3, pp. 683-691, 2009, doi: 10.1109/TEMC.2009.2021258

[11] Y. Lim, S. Wi, J. Kim and Y. Jung, "A Pseudorandom Carrier Modulation Scheme," IEEE Transactions on Power Electronics, vol. 25, no. 4, pp. 797-805, 2010, doi: 10.1109/TPEL.2009.2035699.

[12] T. Jarin and P. Subburaj, "Comprehensive Investigation on Harmonic Spreading Effects of SPWM and RPWM Methods,” European Journal of Scientific Research, vol. 103, no. 2, pp. 296-303, 2013.

[13] L. Mathe, F. Lungeanu, D. Sera, P. O. Rasmussen and J. K. Pedersen, "Spread Spectrum Modulation by Using Asymmetric-Carrier Random PWM," IEEE Transactions on Industrial Electronics, vol. 59, no. 10, pp. 3710-3718, 2012, doi: 10.1109/TIE.2011.2179272.

[14] V. Krishnakumar, V. Kamaraj and S. Jeevananthan, "Random pulse width modulation for performance improvement of brushless DC motor drive," IEEE 2nd International Conference on Electrical Energy Systems (ICEES), 2014, pp. 157-163, doi: 10.1109/ICEES.2014.6924160

[15] K. Kim, Y. Jung and Y. Lim, “A New Hybrid Random PWM Scheme," IEEE Transactions on Power Electronics, vol. 24, no. 1, pp. 192-200, 2009, doi: 10.1109/TPEL.2008.2006613.

[16] H. Li, Z. Li, B. Zhang, F. Wang, N. Tan and W. A. Halang, "Design of Analogue Chaotic PWM for EMI Suppression," IEEE Transactions on Electromagnetic Compatibility, vol. 52, no. 4, pp. 1001-1007, 2010, doi: 10.1109/TEMC.2010.2071878.

[17] P. A. Kumar and N. P. Subramaniam, "Chaotic Triangular Carrier Based Non-Deterministic SPWM Strategy for Voltage Source Inverter Drives," Indian Journal of Science and Technology, vol. 8, no. 9, pp. 842-848, 2015, doi: 10.17485/ijst/2015/v8i9/60075.

[18] F. L. Luo and H. Ye, "Investigation of EMI, EMS and EMC in power DC/DC converters," The Fifth International Conference on Power Electronics and Drive Systems, 2003, pp. 572-577 Vol.1, doi: 10.1109/PEDS.2003.1282904.

[19] S. Zhang, B. Zhang, Q. Lin, E. Takegami, M. Shoyama, and G. Dousoky, "Modeling and Optimization of Impedance Balancing Technique for Common Mode Noise Attenuation in DC-DC Boost Converters," Electronics, vol. 9, no. 480, pp. 1-16, 2020, doi: 10.3390/electronics9030480.

[20] R.W. Erickson and D. Maksimovic, "Fundamentals of Power Electronics Second Edition," Springer, pp. 761-802, 2001.

[21] J. E. Gentel, "Random Number Generation and Monte Carlo Methods," New York:Springer, pp. 1-28, 2005.

[22] S. Perumandla, P. P. Upadhyay and A. J. Laxmi, "Fuzzy based random pulse width modulation technique for performance improvement of induction motor," International Electrical Engineering Congress (iEECON), 2017, pp. 1-4, doi: 10.1109/IEECON.2017.8075735.

[23] Y. Lai, Y. Chang and B. Chen, "Novel Random-Switching PWM Technique With Constant Sampling Frequency and Constant Inductor Average Current for Digitally Controlled Converter," IEEE Transactions on Industrial Electronics, vol. 60, no. 8, pp. 3126-3135, 2013, doi: 10.1109/TIE.2012.2201436.

[24] L. Fakhfakh and A. Ammous, "New simplified model for predicting conducted EMI in DC/DC converters," Electrical Engineering, vol. 99, no. 3, pp. 1087-1097, 2017.

[25] B. Nassireddine, B. Abdelber, C. Nawel, D. Abdelkader and B. Soufyane, "Conducted EMI Prediction in DC/DC Converter Using Frequency Domain Approach," International Conference on Electrical Sciences and Technologies in Maghreb (CISTEM), 2018, pp. 1-6, doi: 10.1109/CISTEM.2018.8613398. 


\section{BIOGRAPHIES OF AUTHORS}
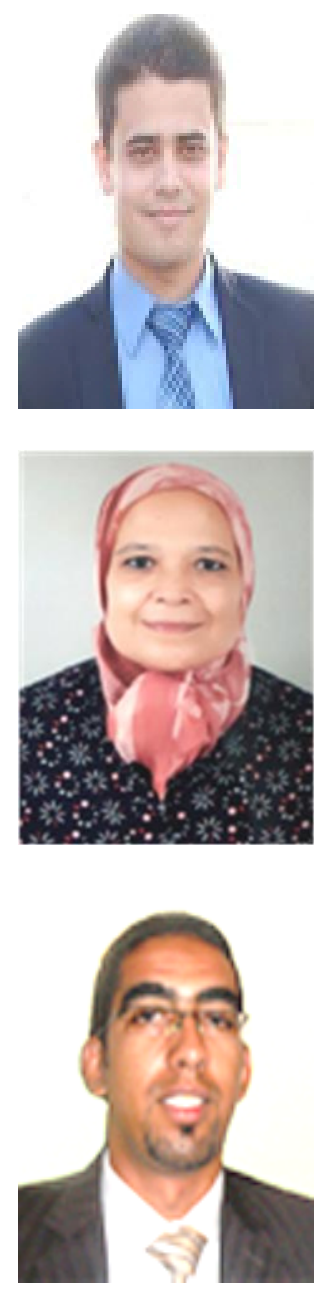

Zakaria M'barki (iD fol SC P was born in Morocco in 1990. He is a $\mathrm{PhD}$ student at the Laboratory of Networks, Computing Science, Telecommunication, and Multimedia (RITM), ESTC Casablanca, Hassan 2 University in Morocco. In 2014, he graduated with honors from Morocco's National School of Electricity and Mechanics with a degree in Electrical and Telecommunication Engineering. His research activities include the development of a control structure capable of optimizing electromagnetic emissions in power electronics converters. $\mathrm{He}$ also has research interests in renewable energy sources, FACTS, power systems, and control system applications. He can be contacted at email: mbarki.ensem@gmail.com.

Kaoutar Senhaji Rhazi (D) SC P \&ualified professor in Electrical Engineering; at the School of Technology in Cacablanca. Morocco. A graduate engineer in electrical engineering from the Mohammadia School of Engineers (EMI) in Rabat. Morocco (in 1991). Had the research preparation certificate (CPR) in telecommunications $\mathrm{PhD}$ in July 2006 (in electromagnetic compatibility). Passed academic qualification in the same field in 2014. Became higher education teacher in 2020. Current reasarch interests are: 'power electronics' and 'electromagnetic compatibility'. She can be contacted at email: senhaji.ksr@gmail.com.

Youssef Mejdoub (iD IS SC P was born in Morocco, in 1980. He received his PhD Thesis on Modeling of Multiconductor Transmission Lines, in 2014 from Cadi Ayyad University, Marrakech Morocco. Since 2016, he has been a Professor at the Superior school of technology (EST), University of Hassan II of Casablanca. He currently works at the Electrical Engineering Department, Superior school of technology. His current research interests is 'antennas', 'electromagnetic compatibility' and 'MTL lines'. He can be contacted at email: ymejdoub@yahoo.fr or youssef.mejdoub@univh2c.ma. 